\title{
An epidemiological investigation of food allergy among children aged 3 to 6 in an urban area of Wenzhou, China
}

Huan Dai ${ }^{1+}$, Fangmin Wang ${ }^{1+}$, Like Wang ${ }^{1}$, Jinyi Wan ${ }^{1}$, Qiangwei Xiang ${ }^{1}$, Hui Zhang ${ }^{1}$, Wei Zhao ${ }^{2}$ and Weixi Zhang $^{1 *}$

\begin{abstract}
Background: The prevalence of food allergy (FA) has increased worldwide. In China, the prevalence of FA in infants and school-aged children is well known, but the prevalence in preschool children is unknown.

Methods: A total of 4151 preschool children aged 3 to 6 years in urban Wenzhou, China, were recruited for this cross-sectional study. Their parents completed a preliminary screening questionnaire, and a detailed FA questionnaire was given to parents whose children had suspected FA according to the preliminary screening. According to the results of the detailed FA questionnaires, some children underwent a skin prick test (SPT) and specific lgE (slgE) measurement. Children with abnormal SPT and/or slgE results who did not meet the diagnostic criteria and those with negative SPT and slgE results whose histories strongly supported FA underwent an oral food challenge (OFC).

Results: Of the 4151 children's parents who completed the surveys, 534 (12.86\%) indicated a positive medical history of FA. Among the 40 children who underwent an OFC, 24 were positive. According to SPT and slgE measurements, 11 children were diagnosed with FA. The prevalence of FA was at least 0.84\%; children who dropped out during the study were considered FA-negative. Among the 35 children with FA, the most common allergic manifestation was skin symptoms. The most common allergic foods were egg, fish and shrimp.

Conclusions: The parent-reported rate of FA in preschool children in urban Wenzhou was $12.86 \%$. The prevalence of FA was at least $0.84 \%$. Among all cases, the most common allergic food was eggs, and the most common allergic manifestation was skin symptoms.
\end{abstract}

Trial registration: NCT03974555, registered on 30 May 2019 (www.clinicaltrials.gov).

Keywords: Food allergy, Preschool, Epidemiology, Prevalence

\footnotetext{
*Correspondence: zhangweixi112@163.com

${ }^{\dagger}$ Huan Dai and Fangmin Wang contributed equally to this work.

1 Department of Pediatric Pulmonology, The Second Affiliated Hospital and

Yuying Children's Hospital of Wenzhou Medical University, 109 Xueyuan

Road, Wenzhou 325027, Zhejiang Province, China

Full list of author information is available at the end of the article
}

(c) The Author(s). 2020 Open Access This article is licensed under a Creative Commons Attribution 4.0 International License, which permits use, sharing, adaptation, distribution and reproduction in any medium or format, as long as you give appropriate credit to the original author(s) and the source, provide a link to the Creative Commons licence, and indicate if changes were made. The images or other third party material in this article are included in the article's Creative Commons licence, unless indicated otherwise in a credit line to the material. If material is not included in the article's Creative Commons licence and your intended use is not permitted by statutory regulation or exceeds the permitted use, you will need to obtain permission directly from the copyright holder. To view a copy of this licence, visit http://creativecommons.org/licenses/by/4.0/ The Creative Commons Public Domain Dedication waiver (http://creativecommons.org/publicdomain/zero/1.0/) applies to the data made available in this article, unless otherwise stated in a credit line to the data. 


\section{Background}

Food allergy (FA) is an adverse reaction to food that is induced by an abnormal or excessive immune response to food allergens. FA includes IgE-mediated, non-IgEmediated (cell-mediated), or mixed (IgE and cellmediated) pathophysiologies [1]. FA manifests as various symptoms, including skin, respiratory, digestive, and cardiovascular symptoms [2]. Following asthma and allergic rhinitis, FA has recently become another allergy epidemic [3]. Worldwide, the prevalence of FA has increased over the last 30 years, with a $6-8 \%$ prevalence rate in children [4]. FA significantly impacts the quality of life of children and their families.

The exact prevalence of FA is difficult to determine because the characteristics of FA differ among races, ages and regions; FA is associated with geographical and dietary differences and countless other unknown factors [5]. The parent-reported rate of FA has been recently increasing as indicated by the epidemiological data of FA. Studies have indicated that the self-reported rate of FA increases by $1.2 \%$ every 10 years [6]. The diagnosis of FA involves obtaining histories (including diet records), a physical examination, a skin prick test (SPT), specific IgE (sIgE) measurement, trial elimination diets and food challenges. The World Allergy Organization (WAO) found that $10 \%$ of countries maintain FA prevalence data based on oral food challenges (OFCs) [7]. According to a study in the United States, in which data were collected by questionnaires and sIgE measurements, the prevalence of FA has increased to $18 \%$ [8]. A study in Australia showed that approximately $10 \%$ of 12 -monthold children were positive for FA, which was confirmed by an OFC [9]. In Thailand, the self-reported rate of FA in preschool children was $9.3 \%$, and the prevalence of FA confirmed by OFC was at least $1.11 \%$ (95\% CI, 0.41$2.98 \%$ ) [10]. In Japan, the prevalence was difficult to obtain, but approximately 350,000 children were diagnosed with FA by doctors [11].

The epidemiology of FA has been extensively studied in other countries and regions, but it has rarely been studied in mainland China. The prevalence of FA and the self-reported rates of FA vary widely. Chen et al. [12] reported that the prevalence rates of $\mathrm{FA}$ in children younger than 2 years old in Chongqing, Zhuhai and Hangzhou were 7.3, 5.8 and 5.6\%, respectively, and a common allergen was eggs. In Guangzhou, a study showed that the self-reported rate of FA in school-age children was $14.6 \%$, and the rate confirmed by SPT and sIgE measurement was $0.31 \%$. Common allergens included shrimp and crab [13].

In China, the FA prevalence in infants and schoolaged children is well known, but the exact prevalence of FA in preschool children is unknown. At 3 to 6 years of age, preschool children are engaging in social experiences for the first time, and both their parents and kindergarten teachers are highly concerned about FA. In the most severe case, FA can lead to anaphylaxis and might result in death within minutes, although it is rare. Therefore, we conducted an epidemiological survey of FA among children aged 3 to 6 years in an urban area of Wenzhou, which will have important scientific and social value.

\section{Methods}

\section{Study design}

Figure 1 shows the flow chart of the study process. This design has been registered in the clinical trials database (ID: NCT03974555). In this study, we selected a random sample of children aged 3 to 6 years from 11 kindergartens in an urban area of Wenzhou, including the Lucheng district, Ouhai district and Longwan district. The study was approved by the medical ethical committee of The Second Affiliated Hospital and Yuying Children's Hospital of Wenzhou Medical University (Project number: LCKY2018-06), and written informed consent was obtained from the parent or legal guardian of the children.

\section{FA questionnaires}

All the participating subjects' parents or guardians were asked to complete the FA screening questionnaire via WeChat. Some parents indicated that their children had diseases or problems caused by certain foods or certain types of food. Researchers ensured that the histories were correct by telephone interviews. Then, the parents were asked to complete the detailed EuroPrevall FA questionnaire [14], and their children underwent medical testing. All the screening questionnaires and detailed questionnaires were checked for completeness by the researchers.

\section{SPT and slgE measurement}

Children who were suspected of having FA underwent SPTs and sIgE measurements after their parents completed the detailed questionnaires. Seventeen allergens including milk, egg white, egg yolk, shrimp, crab, wheat, mackerel, perch, codfish, peanut, cashew, soybean, peach, pineapple, mango, orange and kiwi fruit (Greer Laboratories Inc., America) were used for the SPT. Nine allergens including milk, egg white, shrimp, crab, soybean, peanut, wheat, a mixed nut group and a mixed food group (ALK-Abelló, Denmark) were measured by ImmunoCAP (Phadia25, Phadia AB, Sweden). The mixed food group included egg white, milk, wheat, fish, peanut and soybean.

The SPT results were considered positive if the mean wheal diameter was $3 \mathrm{~mm}$ or greater after subtraction of 


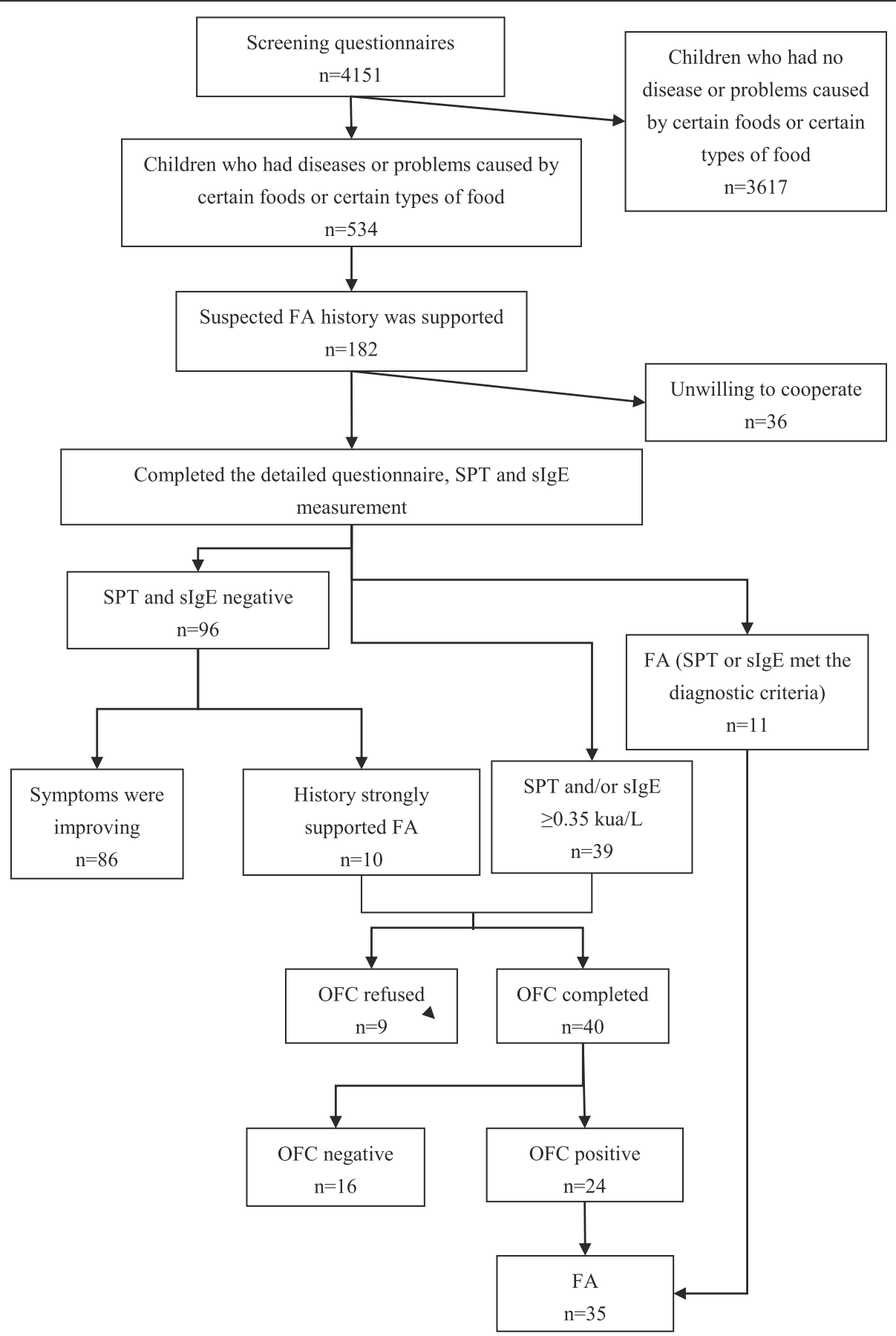

Fig. 1 The flow chart of the food allergy epidemiological investigation

the saline control. Additionally, sIgE measurement results greater than $0.35 \mathrm{kIU} / \mathrm{L}$ were considered positive.

\section{OFC}

Children with abnormal SPT and/or sIgE results who did not meet the diagnostic criteria and those with negative SPT and sIgE results with histories strongly suggestive of FA underwent an OFC. Discontinuation of some medications that may interfere with the OFC may be needed (Table 1) [15].

The OFCs were conducted in the hospital under the supervision of an allergist with close observation of any adverse signs and symptoms, and emergency medicine was prepared because OFC may lead to severe anaphylaxis in some cases. The total amounts of challenge foods administered during the gradually escalating OFC were 
Table 1 Guidelines for discontinuation of medications that might interfere with interpretation of OFC

\begin{tabular}{ll}
\hline Medication & Last dose before OFC \\
\hline Oral antihistamines & $3-10 \mathrm{~d}$ \\
Cetirizine & $5-7 \mathrm{~d}$ \\
Loratadine & $7 \mathrm{~d}$ \\
Oral H2 receptor antagonist & $12 \mathrm{~h}$ \\
Oral long-acting $\beta 2$-agonist & $24 \mathrm{~h}$ \\
Theophylline (liquid) & $24 \mathrm{~h}$ \\
Leukotriene antagonist & $24 \mathrm{~h}$ \\
Oral/intramuscular/intravenous steroids & $3 \mathrm{~d}-2 \mathrm{wk}$ \\
\hline
\end{tabular}

8 to $10 \mathrm{~g}$ of dry food, 16 to $20 \mathrm{~g}$ of meat or fish, and 100 $\mathrm{mL}$ of wet food. Typically, 0.1 to $1 \%$ of the total challenge food was initially administered. The OFC was divided into 4-9 increments, from a minimum dose to a maximum dose; each challenge was administered at a 30-min interval until objective mild symptoms were elicited [15]. Vital signs were monitored before and after the OFC.

\section{Statistical methods}

Data are presented as the rates and means \pm standard deviations. Data were compared using chi-square tests. $P$ values $<0.05$ were considered statistically significant. All statistical analyses were performed using SPSS software.

\section{Results}

The researchers collected 4151 questionnaires, analyzed all information and conducted further investigations and tests as necessary. Table 2 shows the details of parentreported FAs and confirmed FAs in this study.

\section{FA questionnaires and parent-reported FA rate}

A total of 4151 children's parents completed the FA screening questionnaires, resulting in response rates of 52.37 and $47.63 \%$ for males and females, respectively. The average age was $3.99 \pm 0.824$ years. In total, 534 parents indicated that their children had diseases or problems caused by certain foods or certain types of food; therefore, the parent-reported rate of FA was $12.86 \%$. There was no significant difference in the parentreported rate of FA between the sexes (males 284 vs. females 250) in children aged 3 to 6 years in an urban area of Wenzhou $(P>0.05)$. Among the parent-reported cases, the most common allergic food was eggs, and the most common allergic manifestation was skin symptoms.

After telephoning 534 parents or guardians, 352 children were excluded. In one of the 352 children, the symptoms were not caused by food, and another child gradually became food tolerant. A total of 182 children completed medical tests, and their parents completed the detailed FA questionnaire, but 36 dropped out before the end of the study.

\section{SPT and slgE measurement}

SPTs and sIgE measurements were conducted in 146 children. Among the 17 allergens included in the SPT, the allergen with the highest positive rate was shrimp, accounting for $13.01 \%$, followed by crab (12.33\%), eggs (11.64\%), fish (10.96\%) and milk (4.79\%). Among the 9 allergens included in the sIgE measurement, the most common allergen was egg white, accounting for $13.70 \%$, followed by milk (13.01\%), shrimp (8.90\%), the mixed food group (4.79\%) and crab (4.11\%).

According to the results of the SPTs and SIgE measurements, 50 children were positive, and 11 of the 50 children were diagnosed with FA. A total of 96 children were negative according to these two tests.

\section{OFC and the prevalence of FA}

Thirty-nine children with a positive SPT and/or sIgE measurements had abnormal test results but did not meet the FA diagnostic criteria; moreover, 10 children were negative according to the SPT and sIgE measurements, but their histories strongly supported FA. These 49 children were scheduled for an OFC, but the parents or guardians of nine children refused the OFC. Among the 40 children who underwent the OFC, 24 had positive results. Therefore, in this study, 35 children were diagnosed with FA. The prevalence of FA among children aged 3 to 6 years in an urban area of Wenzhou was at least $0.84 \%$ because children who were very likely to have FA and who dropped out during the study were considered negative for FA. In FA-positive children, the most common allergic manifestation was skin symptoms, accounting for $88.57 \%$. The six leading causes of FA were eggs (37.14\%), fish (25.71\%), shrimp (14.29\%), milk (11.43\%), crab (11.43\%) and peanuts (5.71\%).

\section{Discussion}

In this epidemiological investigation, the parent-reported FA rate of preschool children aged 3 to 6 years in an urban area of Wenzhou was $12.86 \%$, and the prevalence of FA was at least $0.84 \%$. Assuming that the likelihood of confirmed FA was equal among the participants and dropouts, the adjusted estimated prevalence of FA in children was approximately $1.36 \%$. This study also indicated that common allergens in preschool children included eggs, fish, shrimp, milk and crab. Skin symptoms were the most common allergic manifestation.

Among Asian countries, the self-reported FA rate in preschool children in Thailand was 9.3\% [10]; a study in Vietnam showed that among children aged 2 to 6 in Hue, the self-reported FA was $9.8 \%$, and in Tien Giang the rate was $7.9 \%$ [16]; additionally, a multi-city study in 
Table 2 The details of parent-reported FA and FA in this study

\begin{tabular}{|c|c|c|c|}
\hline Variable & Total sample & Parent-reported FA(n) & $\begin{array}{l}\text { FA } \\
\text { (n) }\end{array}$ \\
\hline All & 4151 & 534 & 35 \\
\hline \multicolumn{4}{|l|}{ Sex } \\
\hline Male & 2174 & 284 & 24 \\
\hline Female & 1977 & 250 & 11 \\
\hline \multicolumn{4}{|l|}{ Age } \\
\hline$\geq 3$ to $<4$ & 1423 & 181 & 15 \\
\hline$\geq 4$ to $<5$ & 1348 & 193 & 10 \\
\hline$\geq 5$ to $<6$ & 1374 & 159 & 10 \\
\hline$\geq 6$ to $<7$ & 6 & 1 & 0 \\
\hline \multicolumn{4}{|l|}{ Clinical manifestation } \\
\hline Skin rash and pruritus & & 359 & 31 \\
\hline Itching, tingling or swelling in mouth, lips or throat & & 160 & 20 \\
\hline Diarrhoea or vomiting (excluding food poisoning) & & 123 & 12 \\
\hline Sneezing, runny nose or stuffy nose & & 89 & 8 \\
\hline Cough & & 39 & 2 \\
\hline Eyes rubefaction, pain or lacrimation & & 37 & 8 \\
\hline Dyspnoea & & 18 & 3 \\
\hline Others & & 45 & 4 \\
\hline \multicolumn{4}{|l|}{ Food } \\
\hline Milk & & 81 & 4 \\
\hline Egg & & 111 & 13 \\
\hline Shrimp & & 102 & 5 \\
\hline Crab & & 81 & 4 \\
\hline Mango & & 82 & 0 \\
\hline Fish & & 56 & 9 \\
\hline Peanut & & 10 & 2 \\
\hline Others & & 96 & 0 \\
\hline \multicolumn{4}{|l|}{ Allergic history } \\
\hline Eczema & 2035 & 414 & 30 \\
\hline Rhinitis & 1015 & 231 & 17 \\
\hline Wheezing & 368 & 92 & 8 \\
\hline Diagnosed with FA previously & 452 & 281 & 25 \\
\hline
\end{tabular}

China showed that the self-reported FA rate in children aged 3 to 5 years was $6.65 \%$ [17]. Parents may exaggerate the reaction caused by FA because of their anxiety. A disparity was observed between the parent-reported rate and the prevalence of FA among different regions; the prevalence of FA was lower than the parent/self-reported rate. In some developed countries, the FA prevalence in infants was 10\% [7]. A systematic review showed that the FA prevalence rates were similar in children aged 0 to 17 years and adults, at approximately $0.9 \%$ (95\% CI: $0.8-1.1 \%$ ) [18]. The prevalence of FA in preschool children in Thailand was $\geq 1.11 \%$ (95\% CI: $0.41-$
2.98\%) [10]. Children under 3 years old in China had a prevalence rate of 3.5 to $7.3 \%$ [12, 19]. A study in Guangzhou, China, showed that the FA prevalence in children aged 7 to 12 years was $0.31 \%$ [13]; this result was confirmed by SPTs and SIgE measurements. The FA prevalence was higher in this study $(0.58 \%)$ than in the study in Guangzhou. To a certain extent, the FA prevalence in the urban area of Wenzhou is not lower than the prevalence in Guangzhou. The prevalence of FA in this investigation $(0.84 \%)$ was between that of infants and school-aged children in the abovementioned areas. Among the children with FA, 5.71\% were positive for 
multiple allergens; this result was significantly lower than the parent-reported rate of $57.30 \%$. A possible reason is that children may have developed a tolerance to some types of food, such as milk. One study showed that $87 \%$ of children who had milk allergies as infants tolerated milk at 3 years of age [20].

In this study, 534 children had parent-reported FA, and 281 of the 534 children had been diagnosed with FA by doctors, but only 35 children were confirmed to have FA. The number of children who were confirmed to have FA was significantly lower than the number of children who were previously diagnosed with FA by doctors, consistent with the above studies. On the one hand, the parents or guardians in this study had a high level of education and may have had excessive knowledge of FA. They may have interpreted all food-related adverse reactions as FA. In addition, some types of food will gradually become tolerated as children age. On the other hand, the diagnosis of FA still faces the challenges mentioned above, and some parents or guardians refused to complete the detailed questionnaire and consent to the corresponding laboratory tests. To some degree, this result shows the rate of overdiagnosis of FA. The greatest source of misdiagnosis of FA might be the lack of appreciation that a positive test result does not indicate an allergy [1]. Thirty-five children in the study were diagnosed, but only $71.43 \%$ were ever diagnosed by doctors. This result also shows a deficiency in FA diagnosis and indicates that FA need to be diagnosed by a professional allergist. A medical history is crucial for the diagnosis of FA. Several diagnostic methods exist for FA, including SPTs, sIgE measurements, and OFC.

A study showed that SIgE measurements were more sensitive than SPTs in infants; however, in black race, SPTs tended to be more accurate [21, 22]. DunnGalvin et al. [23] used six indices, namely, age, sex, symptoms, SPT results, total IgE (tIgE) results and sIgE results, to predict the clinical diagnosis. They indicated that these six indices had higher sensitivity and specificity than an SPT, sIgE measurement or both. Although the gold standard for FA diagnosis is a double-blinded placebocontrolled food challenge (DBPCFC), in clinical work, an OFC is sufficient to diagnose FA in children. However, an OFC is not commonly performed because of some

Table 3 The cutoff value of SPT and slgE measurement for children over 2 years old

\begin{tabular}{lll}
\hline & slgE $(\mathrm{kua} / \mathrm{L})$ & SPT $(\mathrm{mm})$ \\
\hline Milk & $\geq 15$ & $\geq 8$ \\
Egg & $\geq 7$ & $\geq 7$ \\
Peanut & $\geq 14$ & $\geq 8$ \\
Fish & $\geq 20$ & \\
\hline
\end{tabular}

limitations. SPTs and sIgE measurements are commonly used to diagnose FA, but these results have higher sensitivity, resulting in false positives; therefore, the medical history should be considered along with these results [1]. When clinical history is supported by results of SPTs and/or sIgE measurements at a 95\% PPV, FA is assumed without need for an OFC (Table 3) [15]. There were some children in the study who had a FA history and a positive SPT and (or) sIgE measurement, but the results did not reach the standard. A portion of children had a negative SPT and SIgE measurement, but their history strongly supported FA. These situations require an OFC.

In this study, eggs, fish and shrimp were common allergens in children with FA, while eggs, shrimp and mango were common in parent-reported data. Due to differences in dietary habits, peanuts and wheat have high allergic rates in the United States. In North America and Northern Europe, allergic reactions to fish and shellfish are common, and allergic reactions to Prunoideae fruits are common in the Mediterranean region [24]. In most parts of Asia (China, Korea and some South East Asian countries), egg allergy predominates over cow's milk in children younger than 5 years. Shellfish (crustaceans and mollusks) allergy is the most common food allergy in older children and adults in Asia [25]. Le et al. [16] found that crustaceans are the predominant allergy-inducing food among children aged 2 to 6 in Vietnam. Allergens varied by region even within China. Liu et al. [26] found that the most common selfreported food allergens were eggs among children aged 0-12 months, shrimp among children aged 13-24 months, and fish among children aged 25 to 36 months. In Beijing the main allergen was fruit among children aged 0-14 months [27]. In Guangzhou, shrimp and crab were the most common allergy-inducing foods [12]. Among the patients diagnosed by OFC, the most common allergen in preschool children in Thailand was shrimp [10]. In Chongqing, China, eggs and milk were common allergens among children aged 0 to 1 year with a challenge-proven FA [28]. In Shanghai, the main allergens were eggs, milk, shrimp and fish. Egg allergy was common in children younger than 3 years, and shrimp allergy was common in children older than 3 years [29]. Studies have shown that egg allergy resolves by half at a median age of 74 months, while milk allergy resolves by half at 66 months [30, 31].

The strengths of this study were the large populationbased dataset and the administration of an OFC. This study revealed the parent-reported rate, prevalence rate and clinical features of FA. However, there were some limitations of this study. First, selection bias such as nonresponse bias is unavoidable. Second, the loss to follow-up and the limited allergen test spectrum may have led to some degree of other bias. 


\section{Conclusions}

In this study, the parent-reported rate of FA in preschool children in urban Wenzhou was $12.86 \%$. The prevalence of FA was at least $0.84 \%$. Among all cases, the most common allergic food was eggs, and the most common allergic manifestation was skin symptoms. These results decreased the paucity of FA data in preschool children in China. Further investigations are necessary to explore cross-reactivities between different allergens. Additionally children with FA need to be monitored to improve allergy management. Thus, we are currently working towards these goals.

\section{Abbreviations}

FA: Food allergy; slgE: Specific IgE; OFC: Oral food challenge; SPT: Skin prick test; tlgE: Total IgE; DBPCFC: Double-blinded placebo-controlled food challenge; PPV: Positive predictive value

\section{Acknowledgments}

The authors would like to thank the children participating in this study and their families; the nurses and anyone who contributed to this study.

\section{Authors' contributions}

ZWX and ZW developed the concept and study design. DH, WFM, WLK and WJY collected the data. XQW and ZH performed the experiments. $\mathrm{DH}$ and WFM processed survey data. DH, WFM, WLK, WJY, XQW and ZH performed the statistical analysis. WFM wrote the manuscript. ZWX, ZW and DH edited the final manuscript. The authors read and approved the final manuscript.

\section{Funding}

This investigation is financially supported by Zhejiang Provincial Program for the Cultivation of High-Level Innovative Health Talents, Wenzhou Science and Technology Bureau (Y20180242) and Clinical Research Fundation of The Second Affiliated Hospital and Yuying Children's Hospital of Wenzhou Medical University (SAHoWMU-CR2018-06-214). Zhejiang Provincial Program for the Cultivation of High-Level Innovative Health Talents and Wenzhou Science and Technology Bureau (Y20180242) were involved in the all stages of the study conduct and analysis. Clinical Research Fundation of The Second Affiliated Hospital and Yuying Children's Hospital of Wenzhou Medical University (SAHoWMU-CR2018-06-214) took charge of all costs associated with the development and the publishing of this manuscript.

\section{Availability of data and materials}

The datasets generated during and/or analyzed during the current study are not publicly available due to specific restrictions from the ethics committee, but are available from the corresponding author on reasonable request.

\section{Ethics approval and consent to participate}

The study was approved by the medical ethical committee of The Second Affiliated Hospital and Yuying Children's Hospital of Wenzhou Medical University (Project number: LCKY2018-06). The written informed consent to participate was obtained from the parent or legal guardian of the children.

\section{Consent for publication}

Not applicable.

\section{Competing interests}

The authors declared no competing interests.

\section{Author details}

${ }^{1}$ Department of Pediatric Pulmonology, The Second Affiliated Hospital and Yuying Children's Hospital of Wenzhou Medical University, 109 Xueyuan Road, Wenzhou 325027, Zhejiang Province, China. ${ }^{2}$ Division of Allergy and Immunology, Department of Pediatrics, Virginia Commonwealth University, Richmond, VA, USA.
Received: 12 January 2020 Accepted: 30 April 2020

Published online: 14 May 2020

\section{References}

1. Sicherer SH, Sampson HA. Food allergy: a review and update on epidemiology, pathogenesis, diagnosis, prevention, and management. J Allergy Clin Immunol. 2018;141:41-58.

2. Chinese Medical Association Pediatrics Branch Children's Health Group. Recommendations for diagnosis and treatment of infant food allergy. Chin J Pediatr. 2011:49:344-8.

3. Prescott $\mathrm{S}$, Allen KJ. Food allergy: riding the second wave of the allergy epidemic. Pediatr Allergy Immunol. 2011;22:155-60.

4. Du TG, Foong RM, Lack G. Prevention of food allergy-early dietary interventions. Allergol Int. 2016;65:370-7.

5. National Academies of Sciences, Engineering and Medicine. Finding a path to safety in food allergy: assessment of global burden, causes, prevention, management, and public policy. Washington (DC): National Academies of Sciences, Engineering and Medicine; 2016.

6. Keet CA, Savage JH, Seopaul S, Peng RD, Wood RA, Matsui EC. Temporal trends and racial ethnic disparity in self-reported pediatric food allergy in the United States. Ann Allergy Asthma Immunol. 2014;112:222-9.

7. Prescott SL, Pawankar R, Allen KJ, Campbell DE, Sinn JK, Fiocchi A, et al. A global survey of changing patterns of food allergy burden in children. World Allergy Organ J. 2013;6:1-12.

8. Branum AM, Lukacs SL. Food allergy among children in the United States. Pediatrics. 2009;124:1549-55.

9. Osborne NJ, Koplin JJ, Martin PE, Gurrin LC, Lowe AJ, Matheson MC, et al. Prevalence of challenge-proven IgE-mediated food allergy using population based sampling and predetermined challenge criteria in infants. J Allergy Clin Immunol. 2011;127:668-76.

10. Lao-araya M, Trakultivakorn M. Prevalence of food allergy among preschool children in northern Thailand. Pediatr Int. 2012;54:238-43.

11. Matsubara $Y$, Ae R, Ohya Y, Akiyama H, Imai T, Matsumoto K, et al. Estimated number of patients with food allergy in Japan: the present status and issues regarding epidemiological investigation. Allergy. 2018;67:767-73.

12. Chen J, Liao Y, Zhang ZH, Zhao H, Chen J, Li HQ. Investigation on the status of food allergy among children under 2 years old in three cities. Chin J Pediatr. 2012;50:5-9.

13. Xian $M, L i$ J, Feng $M L$, Pan $Y$, Wei NL. Preliminary investigation on the prevalence of food allergy among school-age children in Guangzhou. Chin J Clinicians. 2013;23:10725-30.

14. Kummeling I, Mills EN, Clausen M, Dubakiene R, Pérez CF, Fernández-Rivas $M$, et al. The EuroPrevall surveys on the prevalence of food allergies in children and adults: background and study methodology. Allergy. 2009;64 1493-7.

15. Nowak-Wegrzyn A, Assa'ad AH, Bahna SL, Bock SA, Sicherer SH, Teuber SS. Work group report: oral food challenge testing. J Allergy Clin Immunol. 2009;123:S365-83.

16. Le TTK, Nguyen DH, Vu ATL, Ruethers T, Taki AC, Lopata AL. A crosssectional, population-based study on the prevalence of food allergies among children in two different socio-economic regions of Vietnam. Pediatr Allergy Immunol. 2019;30:348-55.

17. Xie HL, Shao MJ, Liu CH, Sun ZH, Sha L, Chen YZ, et al. Epidemiology of food allergy in children from 31 cities in China. Int J Pediatr. 2017:44:637-41.

18. Nwaru BI, Hickstein L, Panesar SS. The epidemiology of food allergy in Europe: a systematic review and meta-analysis. Allergy. 2014;69:62-75.

19. Hu Y, Haiqi L. Prevalence of food hypersensitivity in 0-24 months old infants in Chongqing. Chin J Pediatr. 2000;38:431-4.

20. Saarinen KM, Savilahti E. Infant feeding patterns affect the subsequent immunological features in cow's milk allergy. Clin Exp Allergy. 2000;30: 400-6.

21. Du Toit G, Roberts G, Sayre PH, Bahnson HT, Radulovic S, Santos AF, et al. Randomized trial of peanut consumption in infants at risk for peanut allergy. N Engl J Med. 2015;372:803-13.

22. Du Toit G, Roberts G, Sayre PH, Plaut M, Bahnson HT, Mitchell H, et al. Identifying infants at high risk of Peanut allergy: the learning early about Peanut allergy (LEAP) screening study. J Allergy Clin Immunol. 2013;131: 135-43 e1-12

23. DunnGalvin A, Daly D, Cullinane C, Stenke E, Keeton D, ErlewynLajeunesse $M$, et al. Highly accurate prediction of food challenge 
outcome using routinely available clinical data. J Allergy Clin Immunol. 2011;127:633-9 e1-3.

24. Poulsen LK. In search of a new paradigm:mechanisms of sensitization and elicitation of food allergy. Allergy. 2005;60:549-58.

25. Lee AJ, Thalayasingam M, Lee BW. Food allergy in Asia: how does it compare? Asia Pac Allergy. 2013;3(1):3-14.

26. Fangli L, Yibing $N$, Ma D, Zheng Y, Yang X, Wenjun L, et al. Prevalence of self-reported allergy, food hypersensitivity and food intolerance and their influencing factors in 0-36 months old infants in 8 cities in China. Chin J Pediatr. 2013;51:801-6.

27. Shao MJ, Sha L, Liu CH, Zhu WJ, Li S, Luo YQ, et al. Comparison of prevalence of childhood food allergy between urban and rural area in Beijing. Chin J Allergy Clin Immunol. 2017;11:358-64.

28. Chen J, Liao Y, Zhang HZ, Zhao H, Chen J, Li HQ. The prevalence of food allergy in infants in Chongqing, China. Pediatr Allergy Immunol. 2011;22: $356-60$

29. Yang Z, Tongxin C, Wei Z. Clinical analysis of food allergy in 720 children with atopy in Shanghai. J Clin Pediatr Dent. 2009;27:458-61.

30. Sicherer SH, Sampson HA. Food allergy: epidemiology, pathogenesis, diagnosis, and treatment. J Allergy Clin Immunol. 2014;133:291-307.

31. Wood RA, Sicherer SH, Vickery BP, Jones SM, Liu AH, Fleischer DM, et al. The natural history of milk allergy in an observational cohort. J Allergy Clin Immunol. 2013;131:805-12.

\section{Publisher's Note}

Springer Nature remains neutral with regard to jurisdictional claims in published maps and institutional affiliations.

Ready to submit your research? Choose BMC and benefit from:

- fast, convenient online submission

- thorough peer review by experienced researchers in your field

- rapid publication on acceptance

- support for research data, including large and complex data types

- gold Open Access which fosters wider collaboration and increased citations

- maximum visibility for your research: over $100 \mathrm{M}$ website views per year

At BMC, research is always in progress.

Learn more biomedcentral.com/submissions 